\title{
Erratum to: Dihydromyricetin Ameliorates Behavioral Deficits and Reverses Neuropathology of Transgenic Mouse Models of Alzheimer's Disease
}

\author{
Jing Liang • Héctor E. López-Valdés •
}

Hilda Martínez-Coria • A. Kerstin Lindemeyer •

Yi Shen $\cdot$ Xuesi M. Shao $\cdot$ Richard W. Olsen

Published online: 25 June 2014

(c) Springer Science+Business Media New York 2014

Erratum to Neurochem Res (2014) 39:1171-1181

DOI 10.1007/s11064-014-1304-4

In the original publication, the order of authors was listed incorrectly and this has been corrected with this erratum.

The online version of the original article can be found under doi:10.1007/s11064-014-1304-4.

J. Liang $(\bowtie) \cdot$ A. Kerstin Lindemeyer · R. W. Olsen Department of Molecular and Medical Pharmacology, David Geffen School of Medicine, University of California, Los Angeles, Los Angeles, CA 90095, USA

e-mail: jliang@ucla.edu

H. E. López-Valdés

Department of Neurology, David Geffen School of Medicine,

University of California, Los Angeles, Los Angeles, CA, USA

H. Martínez-Coria

Department of Neurobiology and Behavior, University of California, Irvine, CA, USA

Y. Shen

Department of Neurobiology, Zhejiang University School of Medicine, Hangzhou, Zhejiang, China

X. M. Shao

Department of Neurobiology, David Geffen School of Medicine,

University of California, Los Angeles, Los Angeles, CA, USA 